\title{
Implication of conservative and gyroscopic forces on vibration and stability of an elastically tailored rotating shaft modeled as a composite thin-walled beam
}

\author{
Ohseop Song and Nam-Heui Jeong \\ Mechanical Engineering Department, Chungnam National University, Taejon City 305-764, South Korea \\ Liviu Librescu \\ Department of Engineering Science and Mechanics, Virginia Polytechnic Institute and State University, \\ Blacksburg, Virginia 24061
}

(Received 18 October 1999; accepted for publication 4 December 2000)

\begin{abstract}
Problems related with the implications of conservative and gyroscopic forces on vibration and the stability of a circular cylindrical shaft modeled as a thin-walled composite beam and spinning with constant angular speed about its longitudinal axis are addressed. Taking into account the directionality property of fiber reinforced composite materials, it is shown that for a shaft featuring flapwise-chordwise-bending coupling, a dramatic enhancement of both the vibrational and stability behavior can be reached. In addition, the effects played in the same context by transverse shear, rotatory inertias as well as by the various boundary conditions are discussed and pertinent conclusions are outlined. (C) 2001 Acoustical Society of America. [DOI: 10.1121/1.1348301]
\end{abstract}

PACS numbers: 43.40.At [PJR]

\section{INTRODUCTION}

Shaft structural systems rotating about their longitudinal axis are employed for power transmission in aeropropulsion systems, in helicopter drive applications, industrial machines such as steam and gas turbines, turbogenerators, and production lines. In addition, the cutting tools used in boring and milling operations feature similarities in their structural modeling with the shaft systems.

In order to enhance the vibrational behavior, eliminate or even postpone the occurrence of any instability jeopardizing their normal operation, and reduce their weight, advanced structural models have to be devised. In this sense, the integration in their construction of composite materials systems is likely to solve, at least in good measure, the requirement of improved performance of these devices (see Ref. 1).

In addition to their superior strength/weight and stiffness/weight ratios, compared to their metallic counterparts, the directionality property featured by fiber composite material systems can be used to elastically tailor shaft stiffness properties. In such a way, new types of elastic couplings not featured by the standard metallic structures, having beneficial implications upon their response behavior, can be generated. Although belonging to the class of conservative systems, due to the presence of gyroscopic forces, the rotating shaft can behave like a nonconservative system, in the sense that, in some conditions, it can feature divergence and flutter instabilities.

One of the main goals of this study is to put into evidence the conditions under which such instabilities can occur, and the contribution brought about in this regard by the gyroscopic and conservative forces. Moreover, as will be shown, the directionality property of composite materials can be used as to delay the occurrence of both divergence and flutter instabilities, and to enhance the vibrational behavior of the rotating shaft.

The governing equations used in this study incorporate a number of nonclassical features such as transverse shear, rotatory inertias, Coriolis acceleration, and the anisotropy of the spinning structure. Keeping that in mind, a circular cross section of the beam, proper to the shaft structure, is considered; in this case, as will be shown, both the primary and secondary warping functions become zero-valued quantities.

It should be mentioned that problems related with the spinning structures have been approached either in the framework of a solid beam theory (see, e.g., Refs. 2-9), of a circular cylindrical shell theory (see Refs. 10-15), and of the thin-walled beam theory of noncircular cross-section shape (Refs. 16 and 17). However, to the best of the authors' knowledge, with the exception of Refs. 16 and 17 whose results are used herein, no other paper has yet addressed the problems discussed within this work.

\section{COORDINATE SYSTEMS AND BASIC ASSUMPTIONS}

The case of a straight untwisted flexible beam of length $L$ spinning along its longitudinal $z$-axis at a constant rate $\Omega$ and subjected to the longitudinal compressive dead force $P$ is considered (see Fig. 1). Two coordinate systems, an inertial one, $O X Y Z$, and a body attached rotating frame of reference $O x y z$, with their common origin $O$ at the geometric center (coinciding with the elastic center of the beam), are considered. It is supposed that the axes of the two coordinate systems coincide only when $t=0$ while, in the undeformed configuration, the body-fixed and inertial coordinates $\mathrm{Oz}$ and $\mathrm{OZ}$ coincide at any time $t$. Associated with the coordinate systems $(x, y, z)$ and $(X, Y, Z)$, one defines the unit vectors $(\mathbf{i}, \mathbf{j}, \mathbf{k})$ and $(\mathbf{I}, \mathbf{J}, \mathbf{K})$, respectively (Fig. 2). In addition to the previously defined coordinate systems, a local (surface) one, 


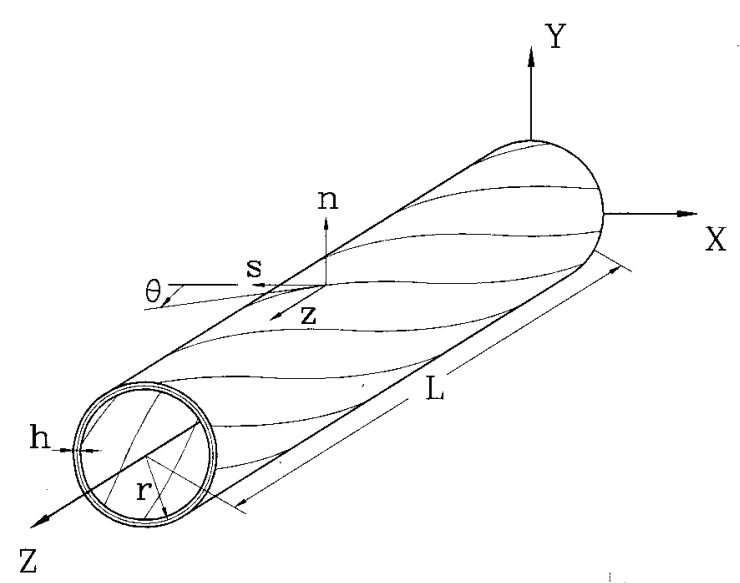

FIG. 1. Composite thin-walled beam of a circular cross section featuring CUS configuration.

$(n, s, z)$, associated with the thin-walled beam, is considered. In light of the stipulated assumptions one can represent the spin rate vector as $\boldsymbol{\Omega}=\Omega \mathbf{k}(\equiv \Omega \mathbf{K})$ with $\dot{\boldsymbol{\Omega}}=0$.

The adopted structural model is that of a thin-walled beam. In this context, the case of a single-cell thin-walled beam of circular cross-sectional shape is considered. Toward its modeling the following assumptions are adopted: (i) the original cross section of the beam is preserved; (ii) transverse shear effects are incorporated; and (iii) the constituent material of the structure features anisotropic properties, and, in this context, a special lay-up inducing flapping-lagging coupling is implemented.

\section{KINEMATICS}

In light of the previously mentioned assumptions, and to reduce the 3D elasticity problem to an equivalent $1 \mathrm{D}$ one, the components of the displacement vector are represented as (see, e.g., Ref. 18)

$$
\begin{aligned}
& u(x, y, z ; t)=u_{0}(z ; t)-y \phi(z ; t), \\
& v(x, y, z ; t)=v_{0}(z ; t)+x \phi(z ; t),
\end{aligned}
$$

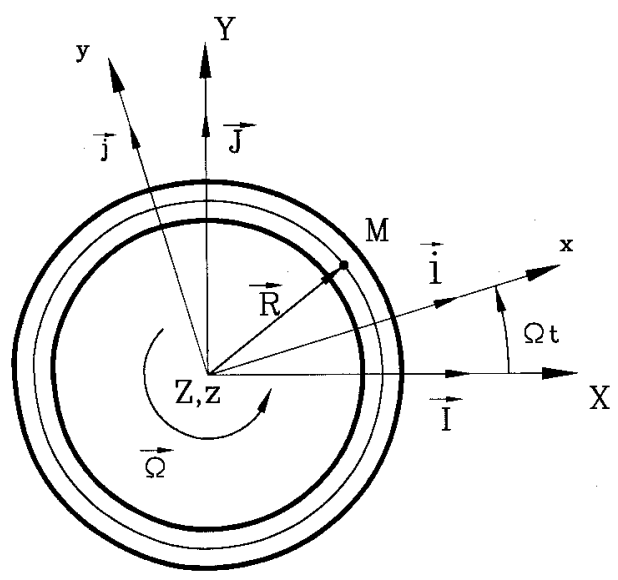

FIG. 2. Inertial $(X, Y, Z)$ and body fixed $(x, y, z)$ coordinate systems.

$$
\begin{aligned}
w(x, y, z ; t)= & w_{0}(z ; t)+\theta_{x}(z ; t)\left[y(s)-n \frac{d x}{d s}\right] \\
& +\theta_{y}(z ; t)\left[x(s)+n \frac{d y}{d s}\right] \\
& -\phi^{\prime}(z ; t)\left[F_{\omega}(s)+n a(s)\right] .
\end{aligned}
$$

In these equations $u_{0}(z ; t), v_{0}(z ; t), w_{0}(z ; t)$ denote the rigid body translations along the $x, y$, and $z$-axes, while $\phi(z ; t)$ and $\theta_{x}(z ; t), \theta_{y}(z, t)$ denote the twist about the $z$-axis and rotations about the $x$ - and $y$-axes, respectively. The expressions of $\theta_{x}$ and $\theta_{y}$ as well as of the geometric quantity $a(s)$ are

$$
\begin{aligned}
& \theta_{x}(z ; t)=\gamma_{y z}(z ; t)-v_{0}^{\prime}(z ; t), \\
& \theta_{y}(z ; t)=\gamma_{x z}(z ; t)-u_{0}^{\prime}(z ; t), \\
& a(s)=-y(s) \frac{d y}{d s}-x(s) \frac{d x}{d s} .
\end{aligned}
$$

In Eqs. (1), $F_{\omega}(s)$ and $n a(s)$ play the role of primary and secondary warping functions, respectively. For their definition see, e.g., Ref. 18. However, for thin-walled beams of circular cross sections, having in view that

$$
\begin{aligned}
& x=-R \sin (s / R), \\
& y=R \cos (s / R), \\
& r_{n}(s)=R,
\end{aligned}
$$

where $R$ is the beam radius of the mid-line cross section, it can readily be proven that both warping quantities exactly vanish, i.e.,

$$
\begin{aligned}
& F_{\omega}(s)=0, \\
& a(s)=0 .
\end{aligned}
$$

It is also seen that in the absence of transverse shear effects

$$
\theta_{x}(z ; t)=-v_{0}^{\prime}(z ; t) ; \quad \theta_{y}(z ; t)=-u_{0}^{\prime}(z ; t) .
$$

In these equations, as well as in those that follow, primes denote differentiation with respect to the longitudinal $z$-coordinate. The position vector of a generic point $M(x, y, z)$ belonging to the deformed structure is

$$
\mathbf{R}(x, y, z ; t)=(x+u) \mathbf{i}+(y+v) \mathbf{j}+(z+w) \mathbf{k},
$$

where $x, y$, and $z$ are the Cartesian coordinates of the points of the continuum in its undeformed state. Recalling that the spin rate was assumed to be constant, and using the expressions for the time derivatives of unit vectors, the velocity and acceleration of a generic point are:

$$
\begin{aligned}
\dot{\mathbf{R}}= & {[\dot{u}-\Omega(y+v)] \mathbf{i}+[\dot{v}+\Omega(x+u)] \mathbf{j}+\dot{w} \mathbf{k}, } \\
\ddot{\mathbf{R}}= & {\left[\ddot{u}-2 \Omega \dot{v}-(x+u) \Omega^{2}\right] \mathbf{i}+\left[\ddot{v}+2 \Omega \dot{u}-(y+v) \Omega^{2}\right] \mathbf{j} } \\
& +\ddot{w} \mathbf{k} .
\end{aligned}
$$

In these equations the superposed dots denote derivatives with respect to time $t$. 


\section{GOVERNING EQUATION SYSTEM}

Toward the goal of deriving the equations of motion of spinning beams and the associated boundary conditions, Hamilton's variational principle is used. This variational principle may be stated as

$$
\begin{aligned}
\delta J= & \int_{t_{0}}^{t_{1}}\left[\int_{\tau} \sigma_{i j} \delta \epsilon_{i j} d \tau-\delta K-\int_{\Omega_{\sigma}} \underset{i}{s_{i}} \delta v_{i} d \Omega\right. \\
& \left.-\int_{\tau} \rho H_{i} \delta v_{i} d \tau\right] d t=0,
\end{aligned}
$$

where

$$
\begin{aligned}
& U=\frac{1}{2} \int_{\tau} \sigma_{i j} \epsilon_{i j} d \tau, \\
& K=\frac{1}{2} \int_{\tau} \rho(\dot{\mathbf{R}} \cdot \dot{\mathbf{R}}) d \tau
\end{aligned}
$$

denote the strain energy and the kinetic energy functions, respectively.

In these equations, $t_{0}$ and $t_{1}$ denote two arbitrary instants of time; $d \tau(\equiv d n d s d z)$ denotes the differential volume element; $s_{i}\left(\equiv \sigma_{i j} n_{j}\right)$ denotes the prescribed components of the stress vector on a surface element of the undeformed body characterized by the outward normal components $n_{i} ; H_{i}$ denotes the components of the body forces; $\Omega_{\sigma}$ denotes the external area of the body over which the stresses are prescribed; $\rho$ denotes the mass density; an undertilde sign identifies a prescribed quantity, while $\delta$ denotes the variation operator. In Eqs. (6) and (7) the Einstein summation convention applies to repeated indices where Latin indices range from 1 to 3 . In the same equations, $\left(v_{1}, v_{2}, v_{3}\right)$ $\equiv(u, v, w), \quad\left(x_{1}, x_{2}, x_{3}\right) \equiv(x, y, z)$.

In light of Eqs. (1), (4), (5), (7), and enforcing Hamilton's condition $\delta v_{i}=0$ at $t_{0}, t_{1}$, it can readily be shown that

$$
\begin{aligned}
\int_{t_{0}}^{t} \delta K d t\left(\equiv-\int_{t_{0}}^{t_{1}} d t \int_{\tau} \rho \ddot{\mathbf{R}} \cdot \delta \mathbf{R} d \tau\right)= & -\int_{t_{0}}^{t_{1}} d t \int_{\tau}\left\{\left[\ddot{u}-2 \Omega \dot{v}-\Omega^{2}(x+u)\right] \delta u+\left[\ddot{v}+2 \Omega \dot{u}-(y+v) \Omega^{2}\right] \delta v+\ddot{w} \delta w\right\} \rho d \tau \\
= & -\int_{t_{0}}^{t_{1}} d t \int_{\tau}\left\{\left[\ddot{u}_{0}-y \ddot{\phi}-2 \Omega\left(\dot{v}_{0}+x \dot{\phi}\right)-\Omega^{2}\left(x+u_{0}-y \phi\right)\right]\left(\delta u_{0}+y \delta \phi\right)\right. \\
& +\left[\ddot{v}_{0}+x \ddot{\phi}+2 \Omega\left(\dot{u}_{0}-y \dot{\phi}\right)-\left(y+v_{0}+x \phi\right) \Omega^{2}\right]\left(\delta v_{0}+x \delta \phi\right)+\left[\ddot{w}_{0}+\left(y-n \frac{d x}{d s}\right) \ddot{\theta}_{x}\right. \\
& \left.\left.+\left(x+n \frac{d y}{d s}\right) \ddot{\theta}_{y}\right] \delta\left[w_{0}+\theta_{x}\left(y-n \frac{d x}{d s}\right)+\theta_{y}\left(x+n \frac{d y}{d s}\right)-\phi^{\prime}\left(\underline{\left.F_{\omega}+n a\right)}\right)\right]\right\} d \tau,
\end{aligned}
$$

where, in light of Eqs. $(2 \mathrm{~g}),(2 \mathrm{~b})$, the terms underscored by a solid line should be discarded.

To induce elastic coupling between flapwise bending and chordwise bending, a special ply angle distribution referred to as circumferentially uniform stiffness (CUS) configuration (see Refs. 18-20), achieved by skewing angle plies with respect to the longitudinal beam axis according to the law $\theta(y)=\theta(-y)$, and $\theta(x)=\theta(-x)$, is implemented. In this case, from the variational principle, Eq. (6), the equations of motion, and the boundary conditions involving this type of coupling are obtained. Employment of constitutive equations and strain-displacement relationships in these equations results in the following governing equations (see Ref. 16):

$$
\begin{array}{cc}
\delta u_{0}: & a_{43} \theta_{x}^{\prime \prime}+a_{44}\left(v_{0}^{\prime \prime}+\theta_{y}^{\prime}\right)-P u_{0}^{\prime \prime} \\
= & b_{1} \ddot{u}_{0}-2 b_{1} \Omega \dot{v}_{0}-b_{1} u_{0} \Omega^{2}, \\
\delta v_{0}: & a_{52} \theta_{y}^{\prime \prime}+a_{55}\left(v_{0}^{\prime \prime}+\theta_{x}^{\prime}\right)-P v_{0}^{\prime} \\
= & b_{1} \ddot{v}_{0}+2 b_{1} \Omega \dot{u}_{0}-b_{1} v_{0} \Omega^{2},
\end{array}
$$

$\delta \theta_{y}: \quad a_{22} \theta_{y}^{\prime \prime}+a_{25}\left(v_{0}^{\prime \prime}+\theta_{x}^{\prime}\right)-a_{44}\left(v_{0}^{\prime}+\theta_{y}\right)-a_{43} \theta_{x}^{\prime}$

$$
=\underline{\left(b_{5}+b_{15}\right) \ddot{\theta}_{y}},
$$

$\delta \theta_{x}: \quad a_{33} \theta_{x}^{\prime \prime}+a_{34}\left(v_{0}^{\prime \prime}+\theta_{y}^{\prime}\right)-a_{55}\left(v_{0}^{\prime}+\theta_{x}\right)-a_{52} \theta_{y}^{\prime}$

$$
=\underline{\left(b_{4}+b_{14}\right) \ddot{\theta}_{x}},
$$

and the boundary conditions at $z=0, L$ :

$$
\begin{array}{ll}
\delta u_{0}: & Q_{x}={\underset{\sim}{Q}}_{x} \text { or } u_{0}={\underset{\sim}{u}}_{0}, \\
\delta v_{0}: & Q_{y}={\underset{\sim}{q}}_{y} \text { or } v_{0}={\underset{\sim}{v}}_{0}, \\
\delta \theta_{y}: & M_{y}={\underset{\sim}{M}}_{y} \text { or } \theta_{y}={\underset{\sim}{\theta}}_{y}, \\
\delta \theta_{x}: & M_{x}={\underset{\sim}{M}}_{x} \text { or } \theta_{x}={\underset{\sim}{\theta}}_{x} .
\end{array}
$$

Herein $Q_{x}(z ; t)$ and $Q_{y}(z ; t)$ denote the shear forces in the $x$ and $y$-directions; $M_{x}(z ; t)$ and $M_{y}(z ; t)$ denote the moments about the $x$-and $y$-axis, respectively; $P$ is the constant axial force, positive in compression, whereas the terms underscored by the dotted and solid lines are associated with Coriolis and rotatory inertia effects, respectively. Using the definitions of shear forces and moments, (see Refs. 16-18), the static version of homogeneous boundary conditions in terms of displacement quantities reads: 


$$
\begin{array}{ll}
\delta u_{0}: & a_{43} \theta_{x}^{\prime}+a_{44}\left(u_{0}^{\prime}+\theta_{y}\right)-P u_{0}^{\prime}=0, \\
\delta v_{0}: & a_{52} \theta_{y}^{\prime}+a_{55}\left(v_{0}^{\prime}+\theta_{x}\right)-P v_{0}^{\prime}=0, \\
\delta \theta_{y}: & a_{22} \theta_{y}^{\prime}+a_{25}\left(v_{0}^{\prime}+\theta_{x}\right)=0, \\
\delta \theta_{x}: & a_{33} \theta_{x}^{\prime}+a_{34}\left(u_{0}^{\prime}+\theta_{y}\right)=0 .
\end{array}
$$

The coefficients $a_{i j}=a_{j i}$ and $b_{i}$ appearing in these equations denote stiffness and reduced mass terms, respectively. Their expressions are displayed in the Appendix. Equations (9) and (11) reveal that in the context of ply angle configuration considered above, in addition to the elastic couplings already mentioned, the flapwise transverse shear is also coupled with chordwise bending, and chordwise transverse shear is coupled with flapwise bending. Moreover, the Coriolis acceleration terms induce a supplementary coupling between the flapwise and chordwise bendings.

Separate from the abovementioned couplings, the extension-twist coupling is induced by the same ply angle configuration. This type of coupling is important and implemented as such, e.g., in helicopter blades and tilt rotor aircraft. However, the present study is confined to only the problem involving the flapwise-chordwise-bending coupling.

\section{TWO REPRESENTATIONS OF GOVERNING EQUATIONS}

Two complex representations of governing equations related to Case (i) and Case (ii) are carried out.

Within Case (i), the system of Eqs. (9) and boundary conditions Eqs. (11) associated with the shearable rotating shaft are used. Keeping in mind that for the circular crosssection beam:

$$
\begin{aligned}
& a_{22}=a_{33} \equiv A, \\
& a_{44}=a_{55} \equiv B, \\
& a_{25}=-a_{34} \equiv S, \\
& b_{4}+b_{14}=b_{5}+b_{15} \equiv C,
\end{aligned}
$$

where $A, B, C$, and $S$ are displayed in the Appendix, and defining the complex displacement variables

$$
\begin{aligned}
& U=u_{0}+i v_{0} ; \\
& \Theta=\theta_{y}+i \theta_{x}, \quad(i=\sqrt{-1})
\end{aligned}
$$

the governing equations can be cast in a complex form as:

$$
\begin{aligned}
& B U^{\prime \prime}+B \Theta^{\prime}+i S \Theta^{\prime \prime}-b_{1} \ddot{U}-2 i b_{1} \Omega \dot{U}+b_{1} U \Omega^{2}=0, \\
& A \Theta^{\prime \prime}-B U^{\prime}-B \Theta-i S\left(U^{\prime \prime}+\Theta^{\prime}\right)-i S \Theta-\underline{C \ddot{\Theta}}=0,
\end{aligned}
$$

while the homogeneous boundary conditions at $z=0, L$ read:

$$
\begin{aligned}
& B\left(U^{\prime}+\Theta\right)+i S \Theta=0 \text { or } U=0, \\
& A \Theta^{\prime}-i S\left(U^{\prime}+\Theta\right)=0 \text { or } U^{\prime}=0 .
\end{aligned}
$$

It is readily seen that the stiffness quantities $B$ and $S$ couple the two governing equations. Solutions of the eigenvalue problem based on this complex representation can be found, e.g., in Refs. 6, 7, and 8.
Case (ii) involves the nonshear deformable beam model. In this case, elimination from Eqs. (9) and (11) of the quantities $a_{44}\left(u_{0}^{\prime \prime}+\theta_{y}^{\prime}\right)$ and $a_{55}\left(v_{0}^{\prime \prime}+\theta_{x}^{\prime}\right)$, an operation followed by consideration of Eq. (3) stating the absence of transverse shear, results in the governing equations:

$$
\begin{aligned}
\delta u_{0}: & a_{22} u_{0}^{\prime \prime \prime \prime}+P u_{0}^{\prime \prime}+b_{1} \ddot{u}_{0}-\underline{\left(b_{5}+b_{15}\right) \ddot{u}_{0}^{\prime \prime}} \\
- & 2 b_{1} \Omega \dot{v}_{0}-b_{1} u_{0} \Omega^{2}=0, \\
& ----- \\
\delta v_{0}: & a_{33} v_{0}^{\prime \prime \prime}+P v_{0}^{\prime \prime}+b_{1} \ddot{v}_{0}-\underline{\left(b_{4}+b_{14}\right) \dot{v}_{0}^{\prime \prime}} \\
+ & 2 b_{1} \Omega \dot{u}_{0}-b_{1} v_{0} \Omega^{2}=0,
\end{aligned}
$$

and of the boundary conditions. Their homogeneous counterpart is:

$$
\begin{aligned}
& a_{22} u_{0}^{\prime \prime \prime}+P u_{0}^{\prime}-\underline{\left(b_{5}+b_{15}\right) \ddot{u}_{0}^{\prime}}=0 \text { or } u_{0}=0, \\
& a_{33} v_{0}^{\prime \prime \prime}+P v_{0}^{\prime}-\underline{\left(b_{4}+b_{14}\right) \ddot{v}_{0}^{\prime}}=0 \text { or } v_{0}=0, \\
& a_{22} u_{0}^{\prime \prime}=0 \text { or } u_{0}^{\prime}=0, \\
& a_{33} v_{0}^{\prime \prime}=0 \text { or } v_{0}^{\prime}=0 .
\end{aligned}
$$

It should be remarked that the governing equations of shearable thin-walled beams Eqs. (9), and their nonshear deformable counterparts, Eqs. (16), exhibit the same order (eight), and as such, in both cases, four boundary conditions have to be prescribed at each end, $z=0, L$, of the beam. Equations (16) reveal that, in this special case, the coupling arises only via the Coriolis acceleration effect. In its absence both equations would be decoupled. However, when this effect is accounted for, upon defining the complex displacement variable $U\left(\equiv u_{0}+i v_{0}\right)$, the governing equation system becomes

$$
A U^{\prime \prime \prime \prime}+b_{1} \ddot{U}-\underline{C \ddot{U}^{\prime \prime}}+P U^{\prime \prime}-b_{1} \Omega^{2} U+2 i b_{1} \Omega \dot{U}=0,
$$

whereas the homogeneous boundary conditions reduce to

$$
\begin{aligned}
& A U^{\prime \prime \prime}+P U^{\prime}-C \ddot{U}^{\prime}=0 \text { or } U=0, \\
& A U^{\prime \prime}=0 \text { or } U^{\prime}=0 .
\end{aligned}
$$

In this case, in order to study the associated eigenvalue problem, a modal analysis can be implemented.

\section{THE EIGENVALUE PROBLEM OF GYROSCOPIC SYSTEMS}

For the cases described by Eqs. (9)-(11), the modal analysis fails to provide a solution to the associated eigenproblem. In contrast to this, in order to find an approximate solution to this problem, assuming synchronous motions, the generalized displacements are represented in the form

$$
\begin{array}{r}
\left(u_{0}(z, t), v_{0}(z, t), \theta_{x}(z, t), \theta_{y}(z, t)\right) \\
=(U(z), V(z), X(z), Y(z)) e^{i \omega t},
\end{array}
$$

where $\omega$ is the complex eigenfrequency, while the spatial parts are represented as 
$(U(z), V(z), X(z), Y(z))$

$$
=\sum_{j=1}^{N}\left(a_{j} u_{j}(z), b_{j} v_{j}(z), c_{j} x_{j}(z), d_{j} y_{j}(z)\right),
$$

where $u_{j}(z), v_{j}(z), x_{j}(z)$, and $y_{j}(z)$ are trial functions which have to fulfil all of the kinematic boundary conditions, whereas

$\mathbf{B}^{T}=\left[a_{1}, a_{2} \cdots a_{N}, b_{1}, b_{2} \cdots b_{N}, c_{1}, c_{2} \cdots c_{N}, d_{1}, d_{2}, \cdots d_{N}\right]$

is a constant vector.

Replacement of the representations in Eqs. (20) in the Hamilton's functional, Eq. (6), carrying out the indicated variations and the required integrations with respect to the spanwise $z$-coordinate and time $t$, and dividing through by $e^{i \omega t}$, the system of governing equations is cast in matrix form as

$$
\delta \mathbf{B}^{T}\left[-\omega^{2} \mathbf{M}_{N}+i \omega \mathbf{G}_{N}+\mathbf{K}_{N}\right] \mathbf{B}=0 .
$$

Herein $\mathbf{M}_{N}, \mathbf{G}_{N}$, and $\mathbf{K}_{N}$ are $(2 N \times 2 N)$ matrices, $\mathbf{M}_{N}$ being symmetric positive definite, $\mathbf{G}_{N}$ skew symmetric, and $\mathbf{K}_{N}$ symmetric. Since contributions from the external compressive load and centrifugal forces are included in $\mathbf{K}_{N}$, its positive definiteness, positive, semidefiniteness, or negative definiteness cannot be posulated ab initio.

Equation (21) provides the following characteristic equation:

$$
\Delta_{N}(\omega)=\operatorname{det}\left[-\omega^{2} \mathbf{M}_{N}+i \omega \mathbf{G}_{N}+\mathbf{K}_{N}\right]=0,
$$

from which $\omega$ can be obtained. If $\mathbf{K}_{N}$ is positive definite and $\mathbf{G}_{N} \neq 0$, the eigenvalues occur as $2 N$ pure imaginary complex conjugate pairs $\lambda_{r}= \pm i \omega_{r}(r=\overline{1,2 N})$, where $\omega_{r}$ is the whirling frequency (see Ref. 21).

For the fixed compressive load, the minimum spin rate at which the whirl frequency becomes zero valued corresponds to the critical spining speed, denoted as $\Omega_{\mathrm{cr}}$. Conversely, for the fixed spin rate, the minimum compressive load for which one of the roots $\omega_{j}$ becomes zero corresponds to the critical compressive load $P_{\text {cr }}$. The instabilities described above are of the divergence type. In general, the condition of divergence is obtained by taking $\omega=0$ in Eq. (22), which yields the equation

$$
\Delta_{N}\left(P, \Omega^{2}\right)=0 .
$$

This equation supplies the divergence instability boundary. On the other hand, combinations of compressive load and spin rate yielding two eigenfrequencies to coalesce constitute a flutter condition. Increasing either of these two parameters beyond the value of the load or spin rate corresponding to the flutter boundary results in complex conjugate eigenvalues, and correspondingly, to bending oscillations with exponentially increasing amplitudes. In the numerical simulations the occurrence of divergence and flutter instabilities will be analyzed for the following three cases of boundary conditions:

(a) Clamped at $z=0: \quad u_{0}=v_{0}=\theta_{y}=\theta_{x}=0$,

Free at $z=L: \quad Q_{x}=Q_{y}=M_{x}=M_{y}=0$,

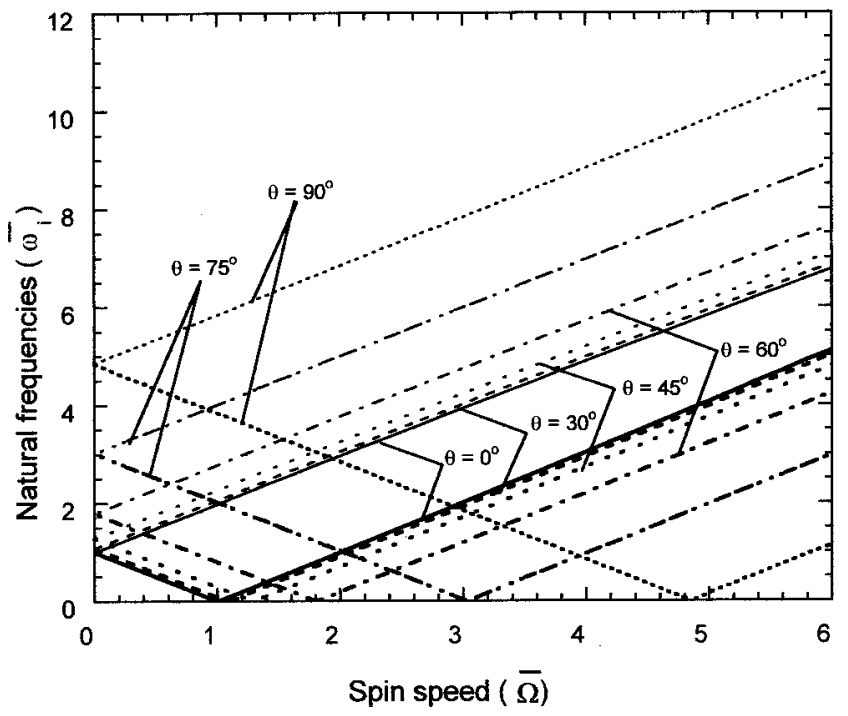

FIG. 3. Variation of upper and lower whirl frequencies with spin speed $\bar{\Omega}$ for selected values of the ply-angle $[\bar{P}=0$, boundary conditions (a)].

(b) Simply supported at both $z=0, L$ :

$$
u_{0}=v_{0}=M_{x}=M_{y}=0 \text {, }
$$

(c) Clamped at both $\mathrm{z}=0, \mathrm{~L}: \quad \mathrm{u} 0=\mathrm{v} 0=\theta \mathrm{x}=\theta \mathrm{y}=0$.

\section{NUMERICAL SIMULATIONS}

In Figs. 3-5, pictorial representations of the variation of natural frequencies $\bar{\omega}_{i}\left(\equiv \omega_{i} / \hat{\omega}\right)$ versus the spin speed $\bar{\Omega}$ $(\equiv \Omega / \hat{\omega})$ for selected ply angles and various boundary conditions, and for the case of the unloaded beam, are displayed. The normalizing factor $\hat{\omega}=138.85 \mathrm{rad} / \mathrm{s}$ is the fundamental frequency of the nonspinning beam counterpart, characterized by $\theta=0^{\circ}$ and $P=0$. The material of the beam is graphite-epoxy whose elastic characteristics are

$$
\begin{aligned}
& E_{1}=30 \times 10^{6} \mathrm{psi}\left(20.68 \times 10^{10} \mathrm{~N} / \mathrm{m}^{2}\right) ; \\
& E_{2}=E_{3}=0.75 \times 10^{6} \mathrm{psi}\left(5.17 \times 10^{9} \mathrm{~N} / \mathrm{m}^{2}\right),
\end{aligned}
$$

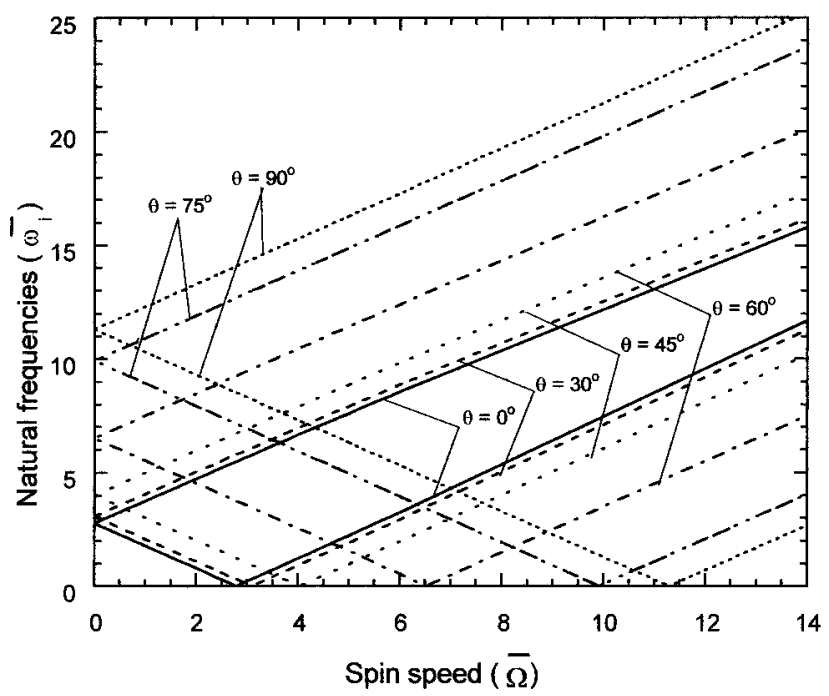

FIG. 4. Counterpart of Fig. 3 for the case of boundary conditions (b). 


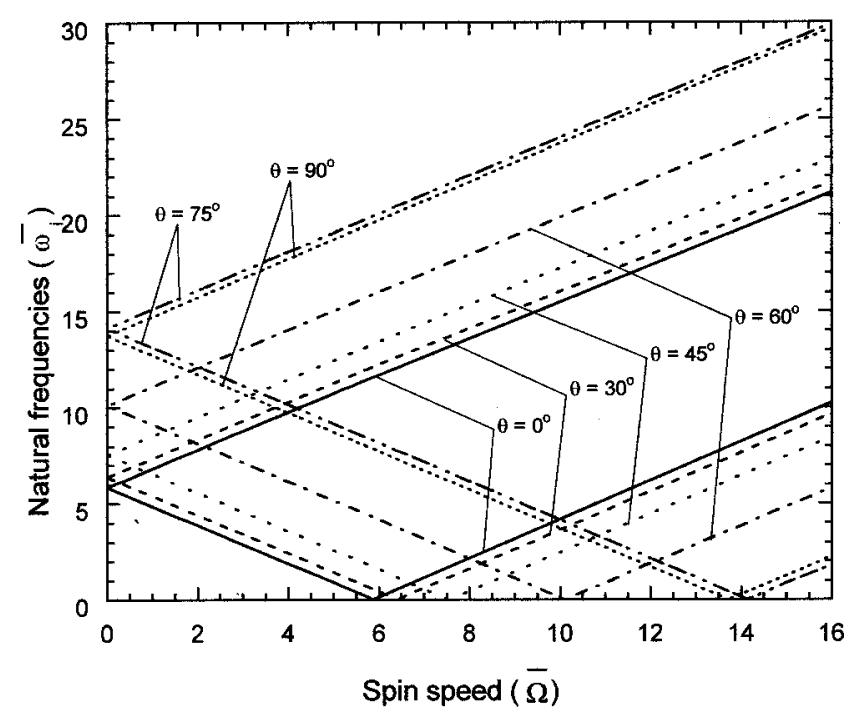

FIG. 5. Counterpart of Figs. 3 and 4 for the case of boundary conditions (c).

$$
\begin{aligned}
& G_{12}=0.45 \times 10^{6} \mathrm{psi}\left(3.10 \times 10^{9} \mathrm{~N} / \mathrm{m}^{2}\right) ; \\
& G_{23}=G_{13}=0.37 \times 10^{6} \mathrm{psi}\left(2.55 \times 10^{9} \mathrm{~N} / \mathrm{m}^{2}\right) ; \\
& \nu_{21}=\nu_{31}=0.00625 ; \quad \nu_{32}=0.25 ; \\
& \rho=0.000143 \mathrm{lb} \mathrm{s}^{2} / \mathrm{in}^{4}\left(1528.15 \mathrm{~kg} / \mathrm{m}^{3}\right) .
\end{aligned}
$$

Concerning the beam geometrical characteristics, these are: $\quad r=5$ in. $(0.127 \mathrm{~m}), \quad L=80 \mathrm{in} .(2.023 \mathrm{~m}), \quad$ and $h$ $=0.4$ in. $\left(0.381 \times 10^{-3} \mathrm{~m}\right)$. For $\bar{\Omega}=0$, i.e., in the case of the absence of gyroscopic effects, it is seen that the system is characterized, for each $\theta$, by a single fundamental frequency. With the increase of the ply angle $\theta$, an increase of nonrotating natural frequencies is obtained. This trend is attributed to the increase of bending stiffnesses $a_{22}=a_{33}(\equiv A)$ associated with the increase of $\theta$ (see, e.g., Ref. 22). As soon as the rotation starts, a fact which is accompanied by the generation of gyroscopic forces, a bifurcation of natural frequencies is experienced. This reverts to the conclusion that due the effect of the gyroscopic Coriolis force, two distinct frequency branches of free bending vibration are produced. The minimum spin rate at which the lowest rotating natural frequency becomes zero valued is called the critical spinning speed, denoted as $\bar{\Omega}_{\mathrm{cr}}$, which corresponds to the divergence instability. Throughout these results it becomes apparent that at each ply angle there is a specific critical spinning speed and that the minimum and maximum ones occur for $\theta=0^{\circ}$ and $\theta=90^{\circ}$, respectively.

The results of these figures also reveal that, for boundary conditions labeled (a), both the critical spinning speed and the eigenfrequencies are, for the same ply angle, larger than those corresponding to boundary conditions (b) and (c), those corresponding to (c) being the least ones. Moreover, Fig. 3 reveals that, for boundary conditions (a), the increase of the ply angle yields a strong and continuous increase of both divergence spin speed $\bar{\Omega}_{\mathrm{cr}}$ and of eigenfrequencies $\bar{\omega}_{i}$. However, for boundary conditions (b) and (c), the results

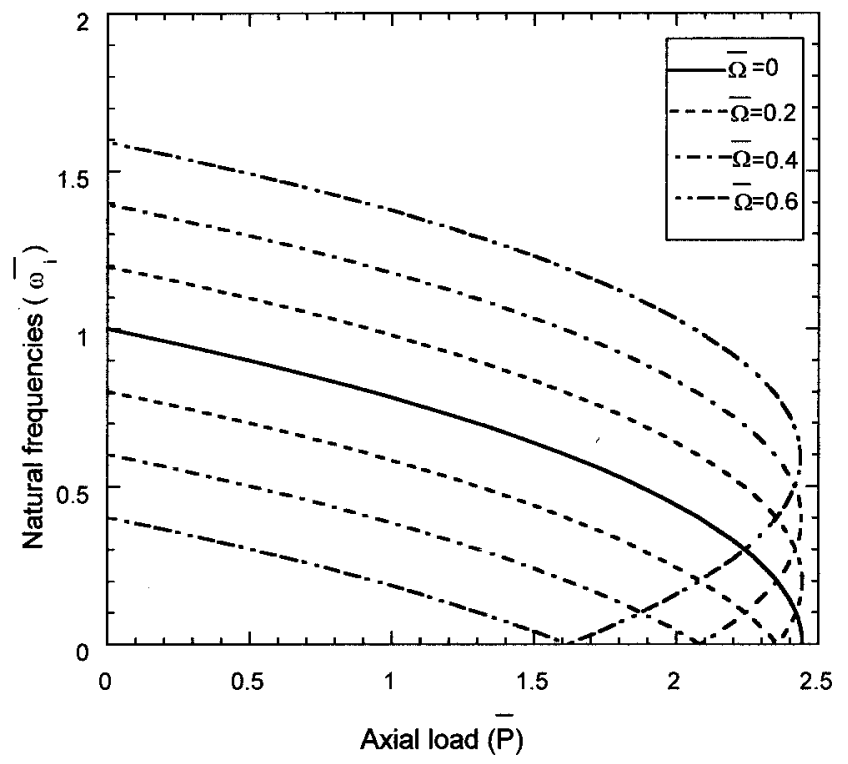

FIG. 6. Variation of upper and lower whirling frequencies with an increase of axial compressive load, for selected values of the spin speed $\left[\theta=0^{\circ}\right.$, boundary conditions (a)].

recorded in Figs. 4 and 5 reveal that, beyond the ply angle, $\theta \approx 75^{\circ}$, much smaller increases of the divergence rotating speed and eigenfrequencies are reached.

Figures 6-9 depict the variation of the upper and lower frequency branches for the boundary conditions (a), and for selected values of the spin rate $\bar{\Omega}$ versus the increase of the dimensionless axial load $\bar{P}\left(\equiv P L^{2} / \hat{a}_{22}\right)$, where $\hat{a}_{22}=1.1787$ $\times 10^{8} \mathrm{lb}$ in. ${ }^{2}$ is the bending stiffness corresponding to the ply angle $\theta=0$. For $\bar{\Omega}=0$, the two frequency branches collapse into a single one. Each of these plots is depicted for a specific value of the ply angle. The results reveal that, for each value of $\bar{\Omega}$, as the axial compressive load increases, the associated eigenfrequency pairs decrease, and for a specific value of $\bar{P}$ these approach the value of zero. The corresponding value of $\bar{P}$ yielding the frequencies to vanish constitutes

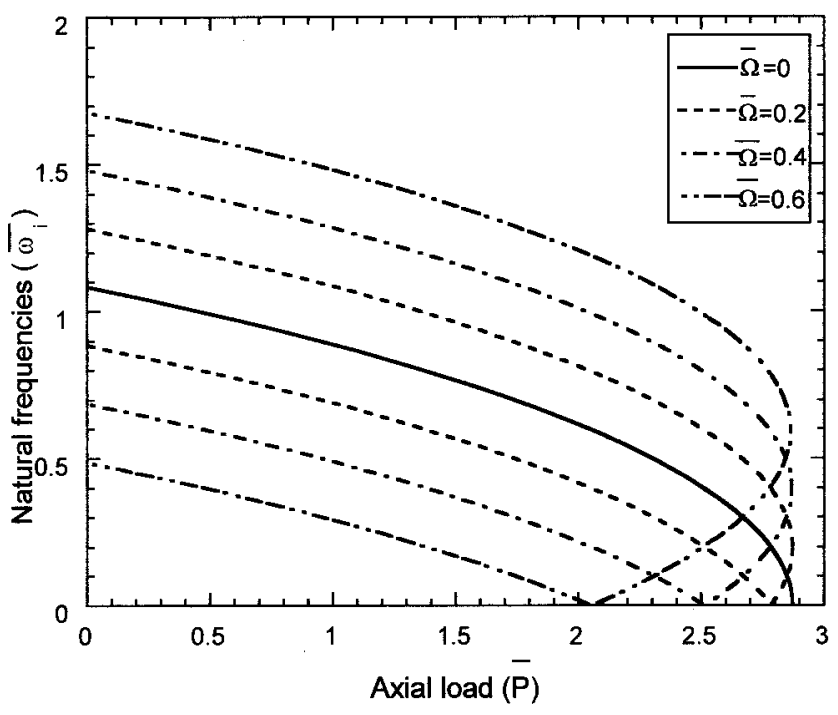

FIG. 7. Counterpart of Fig. 6 for $\theta=30^{\circ}$. 


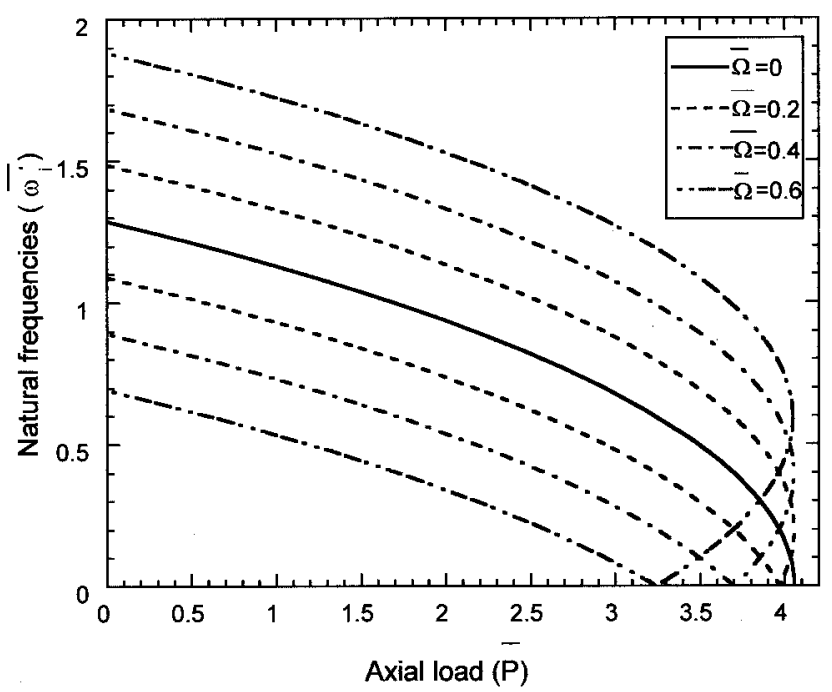

FIG. 8. Counterpart of Fig. 7 for $\theta=45^{\circ}$.

the critical axial compressive load (buckling load). This loss of stability is by divergence. The results of these plots reveal, that for a certain value of the ply angle, the critical axial load, $\bar{P}_{\text {cr }}$, corresponding to $\bar{\Omega}=0$ constitutes an upper bound, in the sense that for $\bar{\Omega} \neq 0$ the critical compressive loads diminish with increasing $\bar{\Omega}$.

However, a closer inspection of the region where the divergence occurs reveals that, for a slightly lower value of the compressive load and for $\bar{\Omega}>0$, the lower and upper lowest eigenfrequencies become complex conjugate. As a result, for $\bar{\Omega}>0$ and the compressive load slightly lower than that corresponding to the divergence instability, the instability by flutter is experienced. A similar trend was also reported in Ref. 23, where the study was accomplished via the finite element method.

The same plots also reveal that the increase of the ply angle results in a remarkable increase of the critical axial load. However, the same plots show that with the increase of the ply angle, the critical axial compressive load becomes

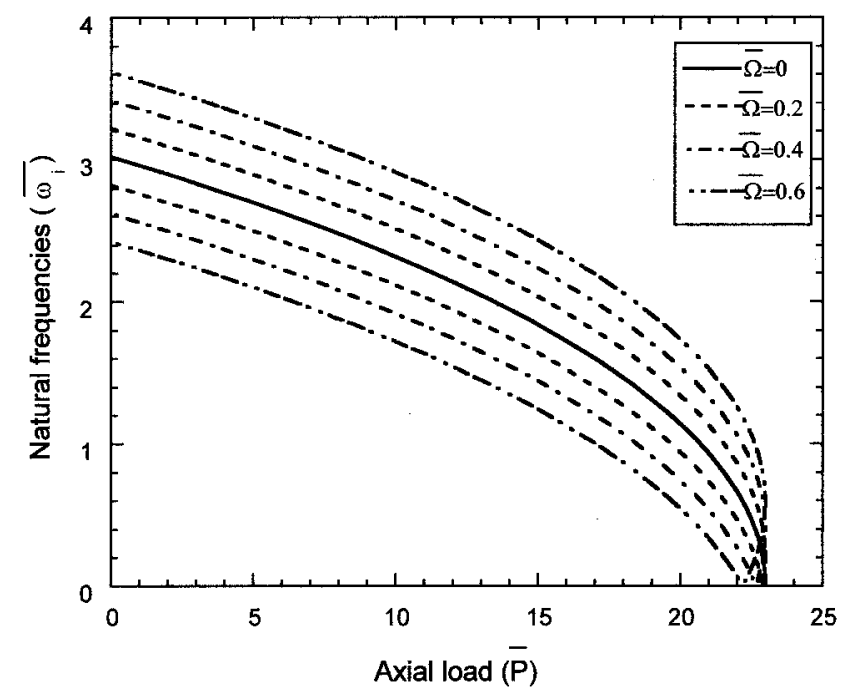

FIG. 9. Counterpart of Fig. 8 for $\theta=75^{\circ}$.

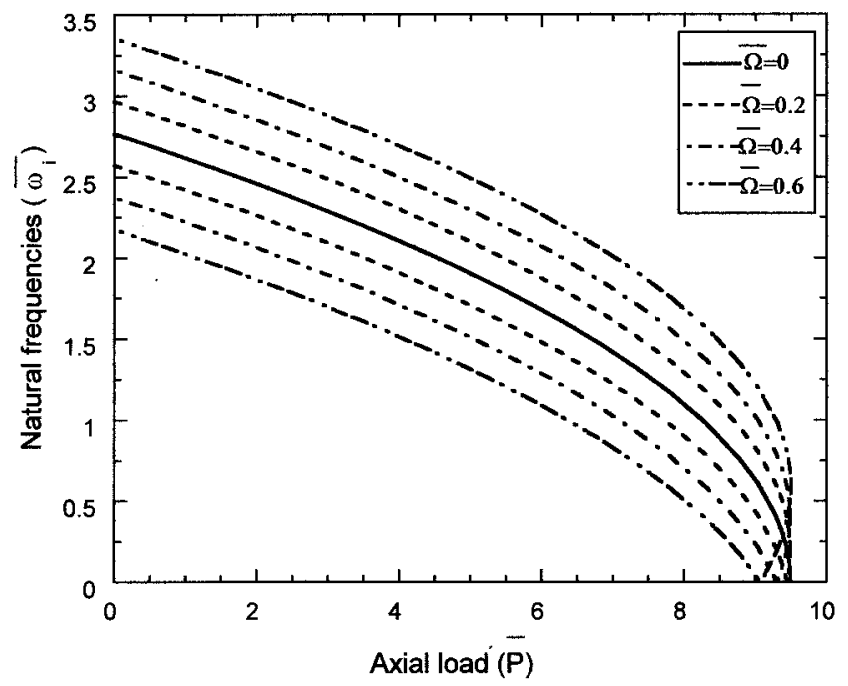

FIG. 10. Variation of upper and lower whirling frequencies with an increase of axial compressive load for selected values of the spin speed $\left[\theta=0^{\circ}\right.$, boundary conditions (b)].

less and less sensitive to the increase of $\bar{\Omega}$. Figures $10-13$ display the counterparts of Figs. 6-9, but generated for boundary conditions (b) and (c), respectively. For these cases, a reduction of the sensitivity of the variation of the buckling load with the spin rate is experienced. This trend becomes more and more prominent in the case of boundary conditions (b) and (c). It should be noticed that, in all of these cases, the tremendous beneficial influence played by the increase of the ply angle toward the increase of the buckling load is remarked.

In Figs. 14-16, stability plots of the spinning system in the $\bar{\Omega}-\bar{P}$ plane for the three cases of boundary conditions (a), (b), and (c) are displayed. In these plots $S$ denotes the region of stability, $D$ denotes the divergence boundary, while $F$ that of the flutter instability. For $\bar{\Omega}$ and $\bar{P}$ equal to zero, $\omega_{i}^{2}$ are real and positive and the system is stable. With the increase of $\bar{\Omega}$ and/or $\bar{P}$, instabilities by divergence or flutter may occur. Due to the fact that the system is conservative,

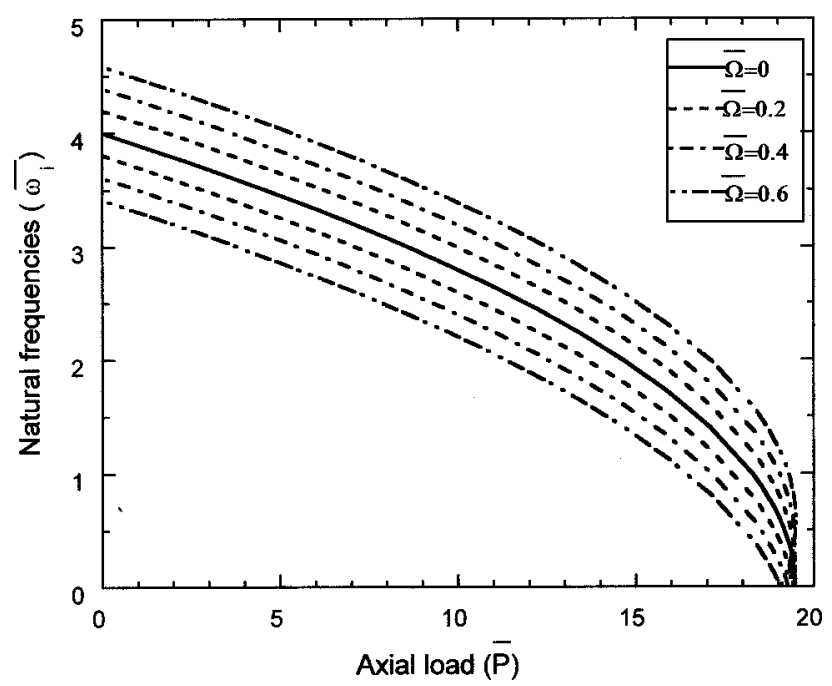

FIG. 11. Counterpart of Fig. 10 for $\theta=45^{\circ}$. 


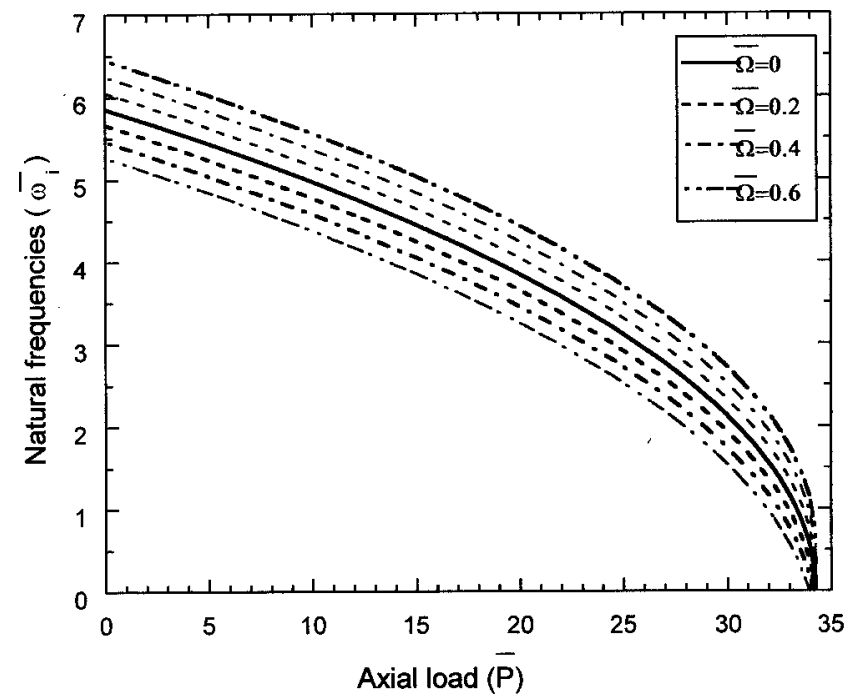

FIG. 12. Variation of upper and lower whirling frequencies with an increase of axial compressive load, for selected values of the spin speed $\left[\theta=0^{\circ}\right.$, boundary conditions $(\mathrm{c})]$.

initial instability will always be of a divergence type, characterized by $\omega_{i}^{2}=0$. The locus of such points in the plane $\bar{\Omega}-\bar{P}$ defines the divergence instability boundary. The plots reveal that in the plane $\bar{\Omega}-\bar{P}$ the instability boundary separates two stable regions, or in other words, divergence occurs only on this boundary, without the existence of regions of divergence instability. In all of these plots, the results reveal that the increase of the ply angle yields a considerable increase of the stability domains. Similar conclusions can be formulated in connection with the implications of boundary conditions, in the sense that as the boundary conditions change from type (a) to types (b) and (c), dramatic increases of the stability domains are experienced. The domain of stability adjacent to the divergence instability boundary and on the right-hand side reveals that the gyroscopic effects, increasing with $\bar{\Omega}$, contribute to the increase of the stability domain. On the same plots the flutter boundary correspond-

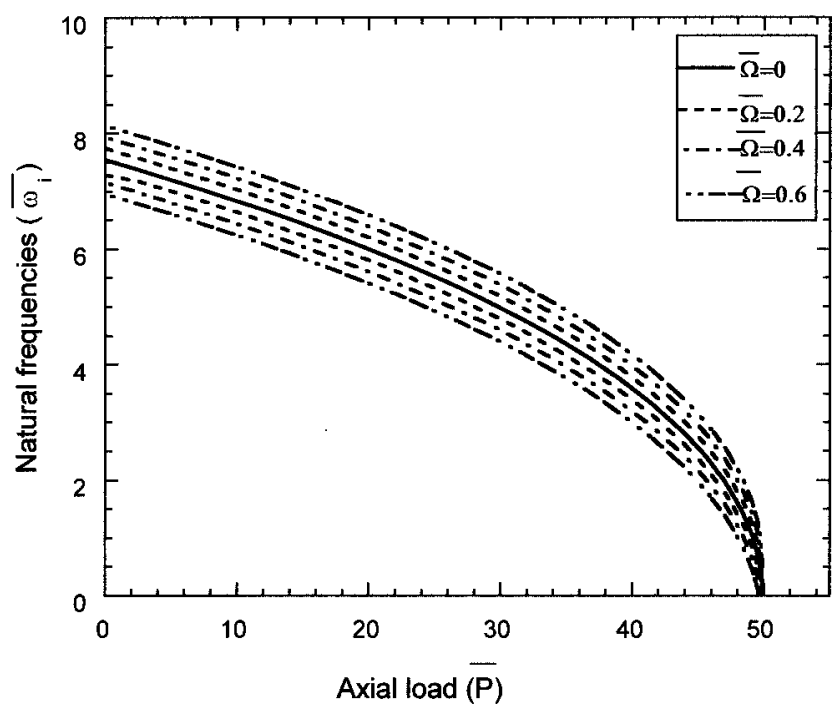

FIG. 13. Counterpart of Fig. 12 for $\theta=45^{\circ}$.

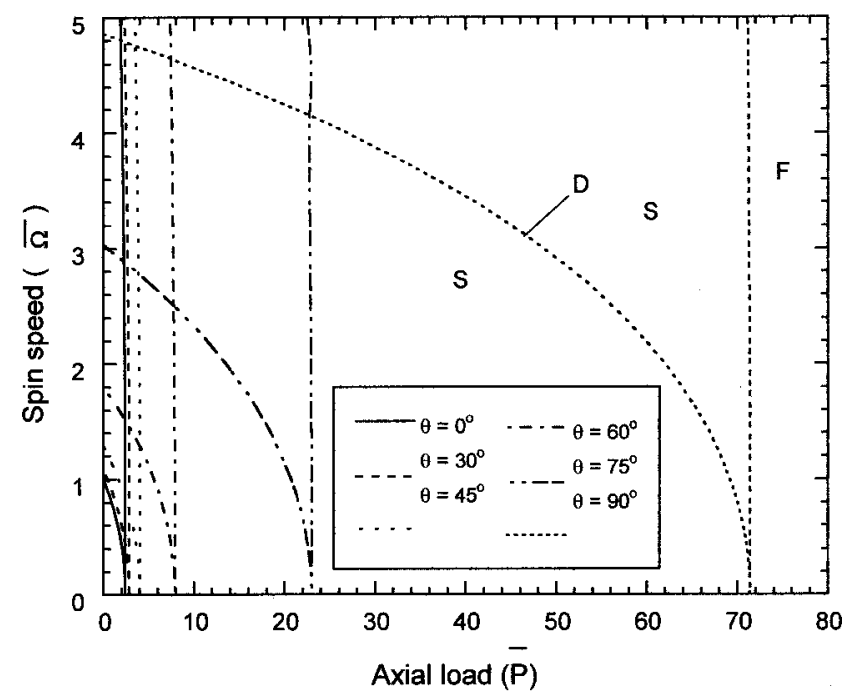

FIG. 14. Stability plot in the $\bar{\Omega}-\bar{P}$ plane displaying the domains of stability, divergence instability boundary, and flutter for selected values of the ply angle [boundary conditions (a)].

ing to conditions involving $\bar{\Omega}$ and $\bar{P}$ yielding coalescence of two frequencies $\omega_{j}^{2}$ is depicted. The results reveal that for specified ply angle and boundary conditions, the flutter boundary consists of slightly curved lines emerging at values of $\bar{P}$ slightly lower than $\bar{P}_{\text {div }}$, and at spin rates $\bar{\Omega}>0$, where $\bar{P}_{\text {div }}$ is the buckling load obtained at $\bar{\Omega}=0$. To have a better view of the flutter and divergence instabilities which appear in a very restrained region of the variation of $\bar{\Omega}$ and $\bar{P}$, Table I displays the trend of variation of the instability boundaries with that of $\theta$ and $\bar{\Omega}$, for boundary conditions (a). The flutter instability domain lies at the right of the curved line $\bar{P}$ $=\bar{P}_{\text {flutter }}$ and for any $\bar{\Omega}>0$. This result coincides with that qualitatively obtained in Refs. 24,25 , with that reported in Ref. 16, and is consistent with that emerging from Figs. 613. However, when the rotatory inertia terms are ignored, in contrast to the abovementioned trend, the flutter boundary does not involve the dependence on $\bar{\Omega}$ and, as a result, in

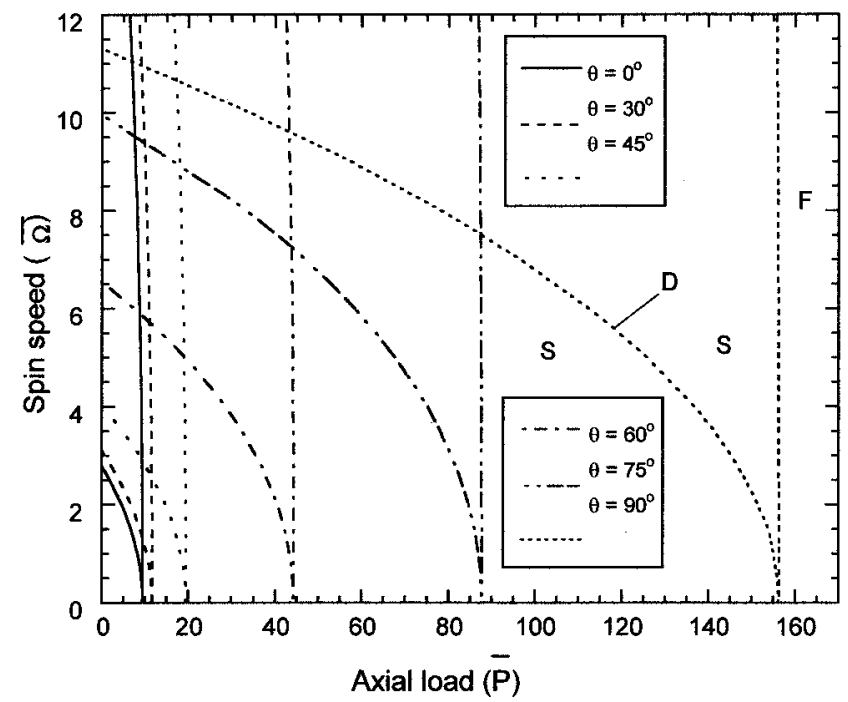

FIG. 15. Counterpart of Fig. 14 for boundary conditions (b). 


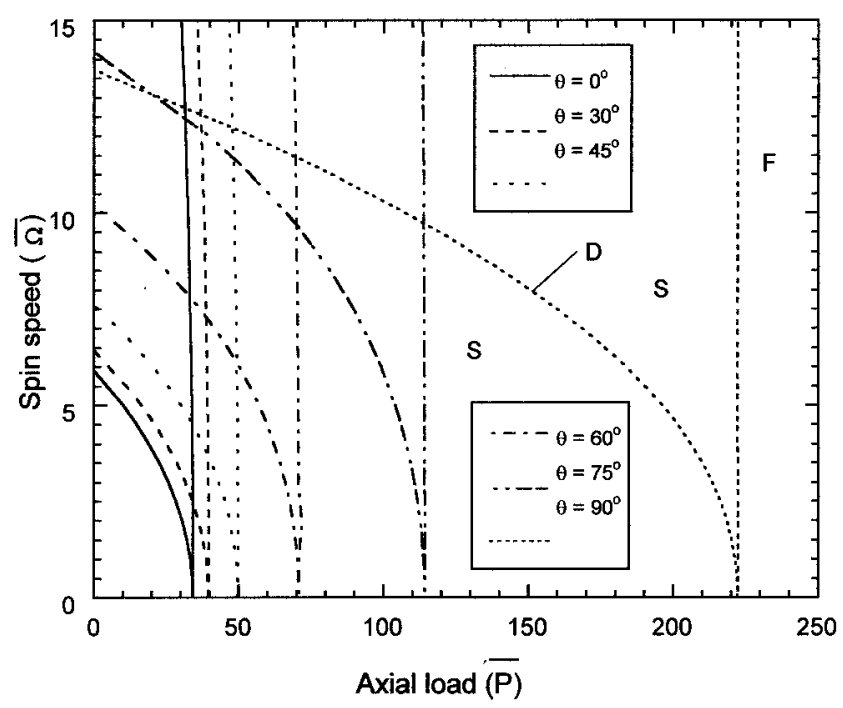

FIG. 16. Counterpart of Figs. 14 and 15 for boundary conditions (c).

that case the flutter boundary degenerates in a straight line $\bar{P}=\bar{P}_{\text {div }}$, parallel to the $\bar{\Omega}$-axis. Such a trend was reported in Refs. 16 and 26.

Finally, in Fig. 17 the effects of transverse shear on the instability boundaries are highlighted. The results reveal that, in the absence of transverse shear effect, i.e, when the structure is unshearable, a significant increase of the stability domain is obtained as compared to the case when transverse shear effect is accounted for. As a result we can say that the classical (i.e., unshearable) structural model inadvertently overestimates the capacity of the rotating shaft to operate without the occurrence of the divergence and flutter instabilities.

\section{CONCLUSIONS}

A study devoted to the vibrational and stability behavior of spinning circular shafts modeled as thin-walled beams was presented, and the implications played by the conservative and gyroscopic forces on their instability behavior have been emphasized. As shown, in the conditions described in the paper, the rotating shaft can experience instabilities by flutter and divergence. Among others, the results reveal that structural tailoring can be successfully employed to enhance their behavior by increasing the spinning speed, and by shifting the domains of divergence and flutter instability toward larger spin rates. The implications of a number of effects,

TABLE I. Stability boundaries for selected values of the ply angle. Boundary conditions (a) and rotatory inertia effects included.

\begin{tabular}{ccccccccc}
\hline \hline & \multicolumn{2}{c}{$\theta=0^{\circ}$} & & \multicolumn{2}{c}{$\theta=45^{\circ}$} & & \multicolumn{2}{c}{$\theta=90^{\circ}$} \\
\cline { 2 - 3 } \cline { 8 - 9 } \cline { 8 - 9 } & $\bar{P}_{\text {div }}$ & $\bar{P}_{\text {fluter }}$ & & $\bar{P}_{\text {div }}$ & $\bar{P}_{\text {flutter }}$ & & $\bar{P}_{\text {div }}$ & $\bar{P}_{\text {flutter }}$ \\
\hline 0 & 2.444 & - & & 4.056 & - & & 71.44 & - \\
1 & - & 2.421 & & 1.713 & 4.033 & & 69.19 & 71.43 \\
2 & - & 2.353 & & - & 3.965 & & 62.02 & 71.39 \\
3 & - & 2.238 & & - & 3.852 & & 48.61 & 71.33 \\
4 & - & 2.078 & & - & 3.692 & & 26.90 & 71.24 \\
5 & - & 1.872 & & - & 3.488 & & - & 71.13 \\
\hline \hline
\end{tabular}

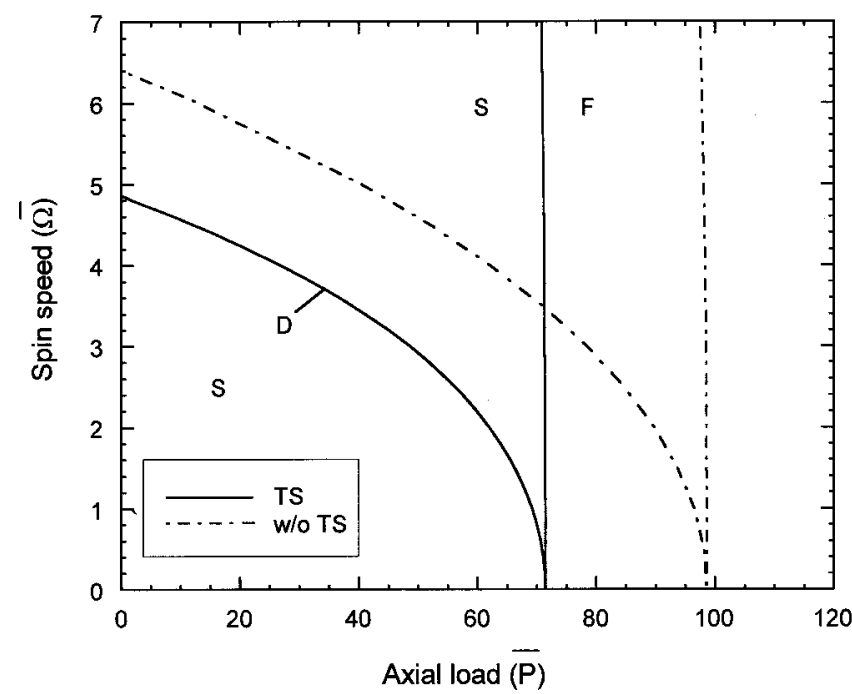

FIG. 17. Effect of transverse shear on the stability boundaries of the rotating shaft [boundary conditions (a); $\theta=90^{\circ}$ ].

such as that of transverse shear upon the stability of the gyroscopic system, have been emphasized. In this context it was shown that discarding transverse shear effect results in an overestimation of the capacity of the system to operate safely, without the occurrence of flutter and divergence instabilities.

Finally, the effects of various boundary conditions on the variation of eigenfrequencies and stability of the system with the spin rate and axial load have been put into evidence.

\section{APPENDIX}

Expressions of stiffness quantities $a_{i j}\left(=a_{j i}\right)$ and reduced mass terms for a cross-section circular shaft:

$$
\begin{aligned}
& a_{22}=a_{33}=\pi R^{2}\left[K_{11}+\frac{2}{R} K_{14}+K_{44}\right] \equiv A, \\
& a_{44}=a_{55}=\pi\left[K_{22}+A_{44}\right] \equiv B, \\
& a_{25}=-a_{34}=-\pi\left[R K_{12}-K_{24}\right] \equiv S, \\
& b_{4}+b_{14}=b_{5}+b_{15}=\pi\left[m_{0} R^{2}+m_{2}\right] \equiv C .
\end{aligned}
$$

Herein

$$
\begin{aligned}
& K_{11}=A_{22}-A_{12}^{2} / A_{11} ; K_{14}=B_{22}-\left(A_{12} B_{12} / A_{11}\right), \\
& K_{12}=A_{26}-A_{12} A_{16} / A_{11}=K_{21}, \\
& K_{44}=D_{22}-B_{12}^{2} / A_{11}, \\
& K_{22}=A_{66}-A_{16}^{2} / A_{11}, \\
& K_{24}=B_{26}-A_{16} B_{12} / A_{11}=K_{42},
\end{aligned}
$$

where $A_{i j}, B_{i j}$, and $C_{i j}$ denote the local stretching, coupling, and bending stiffness components, respectively.

In addition, the mass terms $m_{0}$ and $m_{2}$ are expressed as

$$
\left(m_{0}, m_{2}\right)=\sum_{k=1}^{N} \int_{h_{(k-1)}}^{h_{(k)}} \rho_{(k)}\left(1, n^{2}\right) d n .
$$


${ }^{1} \mathrm{O}$. Bauchau, "Design manufacturing and testing of high speed rotating graphite-epoxy schaft," Ph.D. Thesis, Massachusetts Institute of Technology, Department of Aeronautical and Astronautics, 1981.

${ }^{2}$ C. P. Filipich, M. J. Maurizi, and M. B. Rosales, "Free vibrations of a spinning uniform beam with ends elastically restrained against rotation," J. Sound Vib. 116, 475-482 (1987).

${ }^{3}$ C. W. Lee, R. Katz, A. G. Ulsoy, and R. A. Scott, "Modal analysis of a distributed parameter rotating shaft," J. Sound Vib. 122, 119-130 (1988).

${ }^{4}$ M. L. Chen and Y. S. Liao, "Vibrations of pretwisted spinning beams under axial compressive loads with elastic constraints," J. Sound Vib. 147, 497-513 (1991).

${ }^{5}$ C. W. Bert and C. D. Kim, "Whirling of composite-material driveshafts including bending-twisting coupling and transverse shear deformation," J. Vibr. Acoust. 117, 17-21 (1995).

${ }^{6}$ R. P. S. Han and J. W. Z. Zu, "Modal analysis of rotating shafts. A body fixed axis formulation approach,"' J. Sound Vib. 156, 1-16 (1992).

${ }^{7}$ J. W-Z. Zu and R. P. S. Han, "Natural frequencies and normal modes of a spinning Timoshenko beam with general boundary conditions," J. Appl. Mech. 59, June, 197-204 (1992).

${ }^{8}$ C. L. Liao and Y. H. Dang, "Structural characteristics of spinning pretwisted orthotropic beams," Comput. Struct. 45, 715-731 (1992).

${ }^{9}$ J. W. $\mathrm{Zu}$ and J. Melianson, "Natural frequencies and normal modes for damped spinning Timoshenko beam with general boundary conditions," J. Appl. Mech. 65, 770-772 (1998).

${ }^{10}$ A. Zohar and J. Aboudi, "The free vibrations of a thin circular finite rotating cylinder,' Int. J. Mech. Sci. 15, 269-278 (1973).

${ }^{11}$ S. C. Huang and W. Soedel, "On the forced vibration of simply supported rotating cylindrical shells," J. Acoust. Soc. Am. 84, 275-285 (1988).

${ }^{12} \mathrm{O}$. Rand and Y. Stavsky, "Free vibrations of spinning composite cylindrical shells," Int. J. Solids Struct. 28, 831-843 (1991).

${ }^{13}$ C. D. Kim and C. W. Bert, "Critical speed analysis of laminated composite hollow drive shaft," Composites Eng. 3, 633-643 (1993).

${ }^{14}$ C. W. Bert and C. D. Kim, "Dynamic instability of composite-material drive shaft subject to fluctuating torque and/or rotational speed,' Dynamic and Stability of Systems 10, 125-147 (1995).

${ }^{15}$ L. Hua and K. Y. Lam, "Frequency characteristies of a thin rotating cylindrical shell using the generalized differential quadratic method,' Int. J. Mech. Sci. 40, 443-459 (1998).
${ }^{16} \mathrm{O}$. Song and L. Librescu, "Anisotropy and structural coupling on vibration and instability of spinning thin-walled beams," J. Sound Vib. 204, 477-494 (1998).

${ }^{17} \mathrm{O}$. Song and L. Librescu, "Modelling and vibration of pretwisted spinning composite thin-walled beams," Proceedings of the 38th AIAA/ASME/ ASCE/AHS/ASC Structures, Structural Dynamics, Materials Conference and Exhibition and AIAA/ASME/AHS/Adaptive Structure Forum, Paper AIAA 97-1091, Part 1, pp. 312-322, Kissimmee, Florida, April 7-10 (1997).

${ }^{18}$ O. Song and L. Librescu, "Free vibration of anisotropic composite thinwalled beams of closed cross-section contour,' J. Sound Vib. 167, 129147 (1993).

${ }^{19}$ L. W. Rehfield and A. R. Atilgan, "Toward understanding the tailoring mechanisms for thin-walled composite tubular beams," Proceedings of the First USSR-US Symposium on Mechanics of Composite Materials, May, pp. 187-196 (1989).

${ }^{20}$ E. C. Smith and I. Chopra, "Formulation and evaluation of an analytical model for composite box-beams,' J. Am. Helicopter Soc. 36(3), 23-25 (1991).

${ }^{21}$ L. Meirovitch, Principles and Techniques of Vibration (Prentice Hall, New York, 1997).

${ }^{22}$ L. Librescu, L. Meirovitch, and O. Song, "Refined structural modeling for enhancing vibrational and aeroelastic characteristics of composite aircraft wings," Rech. Aerosp. 1, 23-35 (1996).

${ }^{23}$ D. M. Ku and L. W. Chen, "Stability and whirl speeds of rotating shaft under axial loads," International Journal of Analytical and Experimental Modal Analysis, 9, 111-123 (1994).

${ }^{24}$ K. Huseyin and R. H. Plaut, "Transverse vibrations and stability of systems with gyroscopic forces," Journal of Structural Mechanics 3, 163177 (1974-1975).

${ }^{25} \mathrm{~K}$. Huseyin and R. H. Plaut, "Divergence and flutter boundaries of systems under combined conservative and gyroscopic forces," 1975, Dynamics of Rotors, IUTAM Symposium, Lyngby, Denmark, August 12-16, pp. 182-205 (Springer-Verlag, Berlin, 1974).

${ }^{26}$ R. C. Shieh, "Energy and variational principles for generalized (gyrosopic) conservative problems," Int. J. Non-Linear Mech. 5, 495-509 (1971). 